\title{
PATTERN OF ELECTROLYTE IMBALANCE AND THEIR RESPONSE TO CORTICOSTEROID THERAPY IN STROKE PATIENTS ADMITTED IN A TERTIARY CARE HOSPITAL
}

\author{
AT ISLAM ${ }^{1}, \mathrm{~S} \mathrm{AFROSE}^{2}$, ZK JOARDER ${ }^{3}, \mathrm{KAK} \mathrm{AZAD}^{4}$
}

\begin{abstract}
:
This prospective, single center, case-control study was designed to see the response of electrolyte imbalance in admitted stroke patients receiving corticosteroid therapy in a tertiary care hospital. In IPD, still in many cases of stroke with cerebral edema, injectable corticosteroids are given frequently. Our aim was to find out the common electrolyte imbalances in different type of acute stroke, and, if there was any difference between the patterns of electrolyte imbalance in patients receiving corticosteroids for management, with those who did not receive.

Methods: The source population of this study comprised stroke patients attending the DMCH, between $1^{\text {st }}$ January to $30^{\text {th }}$ June 2014. Total 70 patients, 35 as control and 35 as case group, who received steroids, were randomly enrolled for the study. Detail demographic data were collected from the informant was recorded in structured case report form.

Result: In our study, 48 (68.55\%) patients had infarction and 22 (31.45\%) patients had hemorrhagic stroke. Hyponatremia was detected in majority 51 (72.85\%), others imbalances are hypernatremia 9(12.85\%), hypokalaemia 31(44.28\%), hyperkalaemia 2(2.85\%) and hypochloraemia 37(52.85\%). Out of 48 ischemic stroke, hypernatraemia, hypokalaemia and hypochloraemia was present among 8(16.66\%), 19(39.58\%) and 24(50\%) patients respectively. Out of 22 haemorrhagic stroke patients, 1(4.54\%), 12(54.54\%), 2(9.09\%) and 13(59.09\%) cases were found to have hypernatraemia, hypokalaemia, hyperkalaemia and hypochloraemia respectively. After giving injectable steroids, differences were statistically significant between two group, $p$-value was 0.016 ( $p<0.05)$. Among control group 24(68.57\%); improvement observed was insignificant.
\end{abstract}

Conclusion: It was statistically evident that, patients receiving injectable corticosteroids in acute stroke, showed improvement and had better outcome than of those who did not receive.

Key Words: Stroke, Corticosteroids, Electrolyte Imbalance, Tertiary Care Hospital, Case-control Study.

Received: 02 Novemb er 2016

Accepted: 28 May 2017

\section{Introduction:}

A prospective, single center case-control study that was designed to see the response of electrolyte imbalance in admitted stroke patients receiving corticosteroid therapy (Dexamethasone 5mg IV BD) in a tertiary care hospital. The role of steroids in the management of stroke is still controversial. As stroke is a heterogeneous condition, it is, therefore, unlikely that a single agent would be beneficial in any treatment plan. Perhaps the only general agreement on the use of steroids in stroke is where vasculitis is suspected or proven. As a cause of stroke, vasculitis is very rare ( $<1 \%$ of all strokes), but treatment with steroids should be started whenever it is suspected. ${ }^{1}$

The effectiveness of steroids as one of the options for the treatment of acute stroke, either hemorrhagic or infarction, has never been proven so far. In clinical trials, it is shown that steroid have some role of regulation of plasma osmolarity and ion status regulation. In the management of stroke, in In Patient Department (IPD), still in many cases of suspected cerebral edema, injectable corticosteroids (Dexamethasone $5 \mathrm{mg}$ IV BD) are given frequently. According to Crete-Boston study, intravenous dexamethasone improves outcome in acute

1. Specialist Registrar, Department of Cardiology and CCU, Square Hospital Ltd.

2. Medical Officer, ASHIC Foundation for Childhood Cancer and Palliative Care Unit.

3. Assistant Manager (Medical), Petro-Bangla (GTCL)

4. Professor of Medicine, Dhaka Medical College Hospital.

Correspondence: Dr. Quazi Tamjidul Islam, MBBS, MCPS, FCPS, MRCP (UK), +8801715407784, dr.tamjid@gmail.com

Bangladesh J Medicine 2017; $28: 75-80$ 
hemorrhagic stroke and supports a randomized clinical trial using this approach. ${ }^{2}$ Though the role of corticosteroid still is questionable in reducing cerebral edema, but it may have a role in correction of electrolyte imbalance caused by SIADH, by its salt and water retaining property, ${ }^{3,4}$ which happens in some cases of stroke.

As we know that the corticosteroids have an additional mineralocorticoid like effect as well, the salt and water retention by the steroid may be helpful for minimizing the electrolyte imbalance, along with, reducing the secretion of $\mathrm{ADH}$ and $\mathrm{BNP}$ from the inflamed area of CNS. $3,4,5$

In our study, we tried to find out the common electrolytes status in different type of acute stroke patients and their association with some common clinical presentation. As well as, to find out if there is any difference between the pattern of electrolyte imbalance in patients receiving injectable corticosteroids for management and those who are not receiving.

\section{Methods}

The source population case-control study comprised Stroke patients attending the Dhaka Medical College Hospital, with electrolyte imbalance, with or without receiving steroid (Dexamethasone $5 \mathrm{mg}$ IV BD) were enrolled, until the number of patients getting steroid becomes 35 . Then only stroke with electrolyte imbalance patients without receiving steroid were enrolled until that number becomes 35. Detail demographic data were collected from the informant was recorded in structured case report form. Clinical examination and relevant investigation with $\mathrm{CT}$ scan were done. All collected questionnaire was checked very carefully to identify the error in the data. Data processing work was consisted of registration schedules, editing computerization, preparation of dummy table, analyzing and matching of data. Stroke patient with electrolyte imbalance, includes age, socio-demographic characteristics, clinical manifestation, electrolytes abnormality, and response to corticosteroids therapy. Clinical profile was made by thorough history and clinical examination and by doing some relevant investigation.

\section{Result:}

48 (68.55\%) patients had infarction and 22 (31.45\%) patients had hemorrhagic stroke in our study. Among them, maximum numbers of ischemic stroke were between age group 51-60 (41.42\%), and in case of hemorrhagic stroke maximum numbers were between $61-70(17.14 \%)$ age groups.
Hyponatremia was detected in majority of patients 51 (72.85\%), others imbalance are hypernatremia 9 (12.85\%), hypokalaemia 31 (44.28\%), hyperkalaemia 2 (2.85\%) and Hypochloraemia 37 (52.85\%). Study showed that $32(66.66 \%)$ ischemic stroke patients and $19(86.36 \%)$ hemorrhagic stroke patients had hyponatremia. Out of 48 ischemic stroke, hypernatraemia, hypokalaemia and hypochloraemia was present among 8 (16.66\%), 19 (39.58\%) and 24 (50\%) patients respectively. No hyperkalaemia \& hyperchloraemia recorded among ischemic patients. Out of 22 haemorrhagic stroke patients, was 1 (4.54\%), $12(54.54 \%), 2(9.09 \%)$ and $13(59.09 \%)$ cases were found to have hypernatraemia, hypokalaemia, hyperkalaemia and hypochloraemia respectively. Incidence of hyponatremia was higher in both types of patients, case group 27 (77.14\%) and control group $24(68.57 \%)$. After giving a proper treatment, improvement and responds observed comparatively fine $\&$ greater in the case group patient than the control group. The differences was statistically significant, $p=0.016(p<0.05)$ which may be due to effects of corticosteroids therapy in case group of patients.

Case group 27 (77.14\%) of hyponatremic patients received corticosteroids therapy routinely. Response was significant and recorded in 6 cases. Control group $24(68.57 \%)$ had no steroid therapy; improvement observed was in insignificant fashion and recoded in only 1 patient.

On the other hand clinical or biochemical responses and improvement of hypernatremia was inconsequential and negligible. There is no significant statistical difference between two group, p value $=0.796(\mathrm{p}<0.05)$.

In this study out of 70 patients, 57 patients $(81.42 \%)$ recovered during in hospital stay, 8 patients $(11.42 \%)$ had no noticeable improvement and 5 patients $(7.14 \%)$ had discharged on request or risk bond.

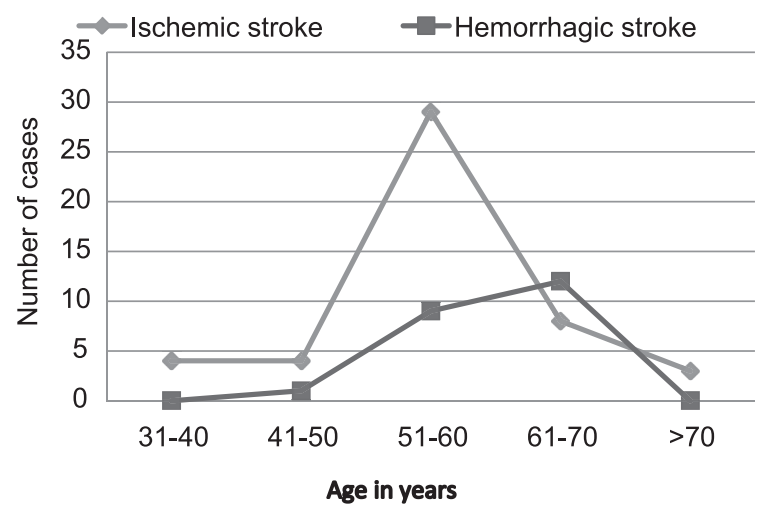

Fig-1 Age distribution of the stroke patients $(n=70)$ 
Table-I

Pattern of electrolyte abnormality in stroke patient.

\begin{tabular}{lcc}
\hline Electrolyte abnormality & $\begin{array}{c}\text { Infarctive stroke } \\
(\mathrm{n}=48)\end{array}$ & $\begin{array}{c}\text { Hemorrhagic stroke } \\
(\mathrm{n}=22)\end{array}$ \\
\hline Hyponatraemia (Serum $\mathrm{Na}^{+}$less than or equal to $\left.135 \mathrm{mEq} / 1\right)$ & $32(66.66 \%)$ & $19(86.36 \%)$ \\
Hypernatraemia (Serum $\mathrm{Na}^{+}$greater than or equal to $145 \mathrm{mEq} / 1$ & $8(16.66 \%)$ & $1(4.54 \%)$ \\
Hypokalaemia (Serum $\mathrm{K}^{+}$less than or equal to $\left.3.6 \mathrm{mEq} / 1\right)$ & $19(39.58 \%)$ & $12(54.54 \%)$ \\
Hyperkalaemia (Serum $\mathrm{K}^{+}$greater than or equal to $\left.5.1 \mathrm{mEq} / 1\right)$ & $24(50 \%)$ & $13(59.09 \%)$ \\
Hypochloraemia (Serum $\mathrm{Cl}^{-}$less than or equal to $\left.95 \mathrm{mEq} / 1\right)$ & 0 & 0 \\
Hyperchloraemia (Serum $\mathrm{Cl}^{-}$greater than or equal to $\left.107 \mathrm{mEq} / 1\right)$ & $0.09 \%$ \\
\hline
\end{tabular}

Table-II

Status of hyponatremia between two groups. $(n=70)$

\begin{tabular}{|c|c|c|c|}
\hline Hyponatraemia & $\begin{array}{l}\text { Case group } \\
\qquad(\mathrm{n}=35)\end{array}$ & $\begin{array}{l}\text { Control group } \\
\qquad(\mathrm{n}=35)\end{array}$ & $\begin{array}{c}\mathrm{p}- \\
\text { value }\end{array}$ \\
\hline At the time of inclusion of study subject & $27(77.14 \%)$ & $24(68.57 \%)$ & 0.016 \\
\hline 4 to 7 days later (with or without steroid therapy) & $21(60 \%)$ & $23(65.71 \%)$ & \\
\hline
\end{tabular}

Table-III

Status of hypernatraemia between two groups. $(n=70)$

\begin{tabular}{|c|c|c|c|}
\hline Hypernatraemia & $\begin{array}{c}\text { Case group } \\
(\mathrm{n}=35)\end{array}$ & $\begin{array}{c}\text { Control group } \\
(\mathrm{n}=35)\end{array}$ & $\begin{array}{c}\mathrm{P}- \\
\text { value }\end{array}$ \\
\hline At the time of inclusion of study subject & $3(8.57 \%)$ & $6(17.14 \%)$ & 0.796 \\
\hline 4 to 7 days later (with or without steroid therapy) & $5(14.28 \%)$ & $4(18.18 \%)$ & \\
\hline
\end{tabular}

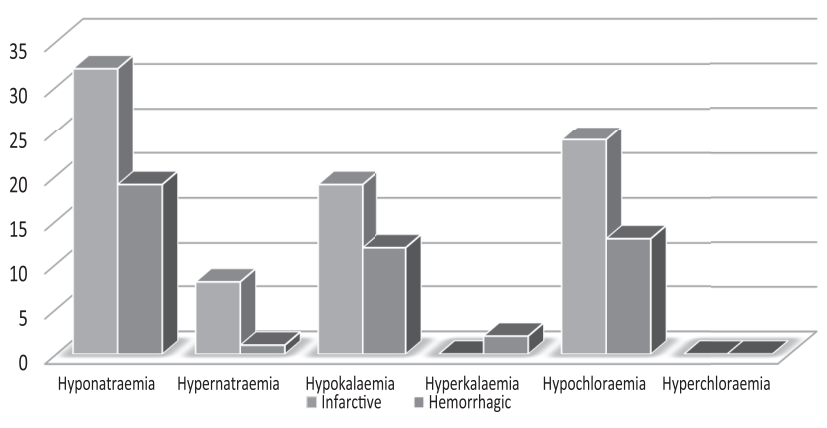

Fig-2 Pattern of electrolyte abnormality in stroke patient.

\section{Discussion:}

Stroke is largely a disease of elderly. In our study peak age incidence was between $5^{\text {th }}$ to $7^{\text {th }}$ decade $(17+21=38)$. Findings correlate with the results of similar studies at home and abroad, e.g. with the results of Hasan MK, Hasan AB, Rubaiyat KA (January 2013). ${ }^{6}$ It is found that the incidence of stroke rose with the increase of age and declined sharply after the age of 70 years, $(8.5 \%)$. This decline

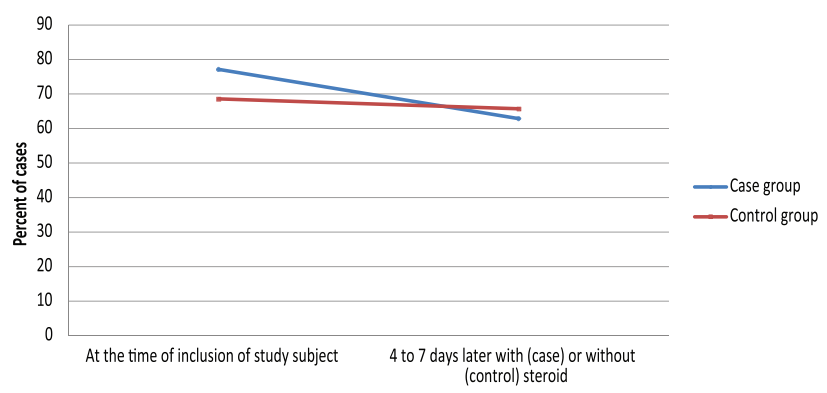

Fig-3 Improvement of hyponatemia (decreased patients number) in comparison between case and control group.

may be due to the fact that in our country life expectancy is lower than those of the developed countries due to the poor health-care facilities.

Patients with clinical diagnosis of haemorrhagic or ischemic stroke, confirmed by CT scan findings, and biochemical evidence of having electrolyte imbalance were enrolled for study. In this study 48 (68.55\%) patients showed infarction and $22(31.45 \%)$ patients showed hemorrhagic stroke. Maximum number of 
ischemic stroke belongs to age group 51-60 (41.42\%), next was age group 61-70 (11.42\%). In case of hemorrhagic stroke maximum number were between 61-70 (17.14\%) age groups and next between 51$60(12.85 \%)$ age group.

$13 \%$ of the stroke patients were found to be diabetic in our study. The study shows that diabetes mellitus is a risk for both types of strokes but it plays more risk for ischaemic stroke. Diabetes mellitus are crucial partner for development and worsening of stroke related hazards and it might increase the patient's morbidity, disability notoriously. Another study ${ }^{7}$ has shown that $10.14 \%$ of stroke patients were diabetic. The Copenhegen stroke study showed that out of 1135 acute stroke patients, 233(20\%) had diabetes mellitus and diabetes influenced stroke in several aspects like age, subtype, speed of recovery and mortality. In a study at BIRDEM ${ }^{8}$ on 165 cases of diabetic patients, all of them developed stroke in less than 10 years of duration of diabetes mellitus. As there is a link, between diabetes mellitus, hypertension and hyperlipidemia, it is difficult to assess its predominant role on stroke. However, when all these conditions are present, the relative risk of electrolyte imbalance from stroke may be greater.

Data on use of steroids in electrolyte disorders in stroke are somewhat scanty and not easily compared. ${ }^{13,14}$ Among the total 70 cases of stroke patients, hyponatremia was detected in majority of patients $51(72.85 \%)$, others imbalance are hypernatremia 9 (12.85\%), hypokalaemia 31 (44.28\%), hyperkalaemia 2 (2.85\%), Hypochloraemia 37 (52.85\%). There are no cases found in Hyperchloraemia. Electrolyte imbalance is one of the major problems in stroke patient. After stroke, either ischemic stroke or haemorrhagic, there are some range of change in body milieu due to nutritional maintenance, immobilization, long time intravenous administration and neuro-hormonal instability. These findings also correlate with other studies. Kusuda K, Saku Y, Sadoshima S, Kozo I, Fujishima M. "Disturbances of fluid and electrolyte balance in patients with acute stroke" 9 showed that the incidences of hypernatremia (serum $\mathrm{Na}$ greater than or equal to $149 \mathrm{mEq} / 1$ ), hyponatremia (less than or equal to $134 \mathrm{mEq} / \mathrm{l}$ ), hyperkalemia (serum $\mathrm{K}$ greater than or equal to 4.8 $\mathrm{mEq} / \mathrm{l}$ ) and hypokalemia (less than or equal to 3.2 $\mathrm{mEq} / 1)$ were higher in patients with hemorrhage (18, 7,13 and $14 \%$, respectively) than infarction $(4.5,4.5$, 11 and $6 \%$, respectively). The incidences of hypernatremia and hyponatremia in infarction were higher in those who had cortical lesions than in those who had lesions in the basal ganglia or infratentorium. ${ }^{11}$ In cerebral hemorrhage, the incidence of hypernatremia was the highest in those with brain stem lesion. Hypernatremia was found in $27 \%$ of large sized hematoma, being significantly higher than that of those with medium (16\%) or small (1\%) hematoma. A similar tendency was also observed in hyponatremia and hyperkalemia. In elderly patients, electrolyte disturbances were more common than in young or middle-aged patients. Renal insufficiency and diabetes mellitus were frequent complications in stroke patients with hypernatremia (42 and $32 \%$, respectively), of which $57 \%$ died within one month of admission. Findings are quite different due to study design $\&$ methodology in their study ${ }^{10}$ with our hospital management system, inadequate patients care, indiscriminate drug use and poor nutritional support in our country.

Our study shows that 19 (66.66\%), 32 (86.36\%) had hyponatremia in ischemic, hemorrhagic stroke respectively. Out of 48 ischemic stroke it was 8 (16.66\%), 19 (39.58\%) and 24 (50\%) Hypernatraemia, Hypokalaemia and Hypochloraemia respectively. No Hyperkalaemia \& Hyperchloraemia recorded in ischemic patients. Out of 22 haemorrhagic stroke patients was 1 (4.54\%), 12 (54.54\%), 2 (9.09\%) and 13 (59.09\%) Hypernatraemia, Hypokalaemia, Hyperkalaemia and Hypochloraemia respectively. No Hyperchloraemia found in hemorrhagic patients.

Incidence of hyponatremia was higher in both types of patients, case group 27 (77.14\%) and control group $24(68.57 \%)$. After giving a proper treatment, improvement and responds observed comparatively fine $\&$ greater in the case group patient than the control group. The differences was statistically significant between two group, p-value was 0.016 $(p<0.05)$. It may be due to effects of corticosteroids therapy in case group of patients. On the other hand clinical or biochemical responds, improvement of hypernatremia was inconsequential and negligible. There is no significant statistical difference between two group $(\mathrm{p}=0.796)$.

Among 70 patients, 51 patients had hyponatremia. All patients evaluated \& managed routinely. Considerable change and difference observed between case and control groups on the relation with steroids therapy. Case group 27 (77.14\%) of hyponatremic patients received corticosteroids therapy routinely. Responds were significantly occurred and recorded 6 cases. Following improvement patient number reduced 21 (60\%). Control group 24 (68.57\%) had no steroid therapy; improvement observed in insignificant fashion and recoded only 1 patient. The ratio between case and control groups 6:1. 
Gradual communication of the truth within the context of continued support and encouragement almost always leads to enhanced hope. ${ }^{12}$ Breaking bad news generally causes distress to both the patient and his/her family. In this issue we obtain the response from the attendance by consultation, counseling, open question and elicit the feelings, emotion and concern. We found that only $13 \%$ of attendance carries clear idea regarding the disease, but majority of patient attendance lack of concept about the disease, its management and prognosis on future time.

Patients symptoms, degree of disability or dependence in the daily activities and clinical outcome had evaluated and measured by modified Rankin Scale (MRS). Study shows that $57(81.42 \%)$ of the patients recovered either completely or partially (MRS score 0 to 2$), 8(11.42 \%)$ remains standstill and they discharged on request or risk bond (MRS score 3 to $5) .5$ cases $(7.14 \%)$ expired during hospital stay (MRS score 6).

\section{Conclusions \& recommendations:}

Stroke is a complex disease that requires the efforts and skills of all members of the multidisciplinary team. A coordinated care of the stroke patient results in improved outcome, decreased length of stay, and decreased costs. Stroke patient die of either due to the primary disease or due to complications. Medical management focuses on the prevention of sub-acute complications of stroke, including malnutrition, aspiration, pneumonia, electrolyte Imbalance, UTI, bowel or bladder dysfunction, DVT, pulmonary embolism, contractures, joint abnormalities, and skin breakdown. Electrolyte disturbances such as hypernatraemia or hyponatraemia, resulting from the syndrome of inappropriate antidiuretic hormone (SIADH), increase of brain natriuretic peptides (BNP), inappropriate fluid intake and loss, can lead to complications such as seizures or death. Complications like dyselectrolytaemia are more common in acute phase.

In our country, there are many studies on stroke, its associated conditions and their effect on stroke patients' outcome, but a few studies on electrolytes disturbance in stroke patients have been done, in home as well as abroad, so far. Hence, we undertook this study with the aim to see the pattern of common electrolyte disturbance to be found in IPD stroke patient, as well as to see, whether there is any change in the electrolyte pattern in those who were receiving injectable corticosteroids (Dexamethasone $5 \mathrm{mg}$ IV $\mathrm{BD})$.
Finally, in our study, it was statistically evident that, patients receiving injectable corticosteroids (Dexamethasone 5mg IV BD), showed improvement and had better outcome than of those who were not.

But the final clinical judgment call to give IV steroid should be made by the assessing physician after clinical assessment of the patient's condition as there are lots of variable factors associated with the pathogenesis and disease progression in case of stroke.

\section{Limitations of the study}

This is a small study; only patients of stroke admitted in Dhaka Medical College Hospital (DMCH) were taken for the study. So this will not reflect the overall picture of the country. A large scale study needs to be conducted to reach to a definitive conclusion.

Majority of the patients were poor and illiterate. Many had no proper treatment, checkup. Patient comes in tertiary center after development of some hazards, complication. So some hazards, risk factors are subsistence before and after treatment.

Sample were taken by purposive method in which question of personal biasness might arise.

\section{References}

1. Zuber M. Isolated angiitis of the central nervous system. In: Bogousslavsky J, Caplan L, eds. Uncommon Causes of Stroke. Cambridge, UK: Cambridge University Press; 2001: 1-9.

2. Zaganas I, et al. A comparison of acute hemorrhagic stroke outcomes in 2 populations: the Crete-Boston study.Stroke. 2011 Dec;42(12):3640-2. doi: 10.1161/STROKEAHA.111.632174. Epub 2011 Oct 20.

3. Katayama Y, et al. A randomized controlled trial of hydrocortisone against hyponatremia in patients with aneurysmal subarachnoid hemorrhage.Stroke. 2007 Aug; 38(8):2373-5. Epub 2007 Jun 21.

4. Moro N, et al. Prophylactic management of excessive natriuresis with hydrocortisone for efficient hypervolemic therapy after subarachnoid hemorrhage.Stroke. 2003 Dec;34(12):2807-11. Epub 2003 Dec 1.

5. "The top 10 causes of death". WHO.

6. Hasan MK, Hasan AB, Rubaiyat KA: Electrolyte Disturbances in Acute Phase of Stroke Patients. 2013 Jan; 6 (1):12-16

7. Zahed Uddin "electrolyte imbalance in acute stroke patients" (January 2013)

8. Latif ZA. Zaman SM, Ahad A, Rahim SA. Study of stroke between normotensive and hypertensive NIDDM cases in BIRDEM, Dhaka, BANGLADESH, J Neurosci.1990;6: 52-59. 
9. Kusuda K, Saku Y, Sadoshima S, Kozo I, Fujishima M. "Disturbances of fluid and electrolyte balance in patients with acute stroke"; 1989 May;26(3):223-7.

10. Langhorne P, Stott DJ, Robertson L, et al. Medical complications after stroke: a multicenter study. Stroke 2000;31:1223-29

11. Mannan and Alamgir, and Chowdhury et al. Study of stroke among the hospital admitted patients with assessment of cerebral perfusion and arterial anatomy in correlation with CT: early clinical results. 1998; 19:29
12. Stroke Management Guideline(Draft) 2008. Directorate General of Health Services Mohakhali, Dhaka.

13. Feigin VJL, Lawes CMM, Bennett DA, Anderson CS. Stroke epidemiology: a review of population-based studies of incidence, prevalence, an case-fatality in the late 20th century. Lancet Neurol. 2003; 2:43-53.

14. Qizilbash N, Lewington SL, Lopez-Arrieta JM. Corticosteroids for acute ischemic stroke (Cochrane Review) The Cochrane Library, Issue 1, 2003. Oxford: Update Software; 2003. 